\title{
INTEGRAL p-adic NORMAL MATRICES SATISFYING THE INGIDENGE EQUATION
}

\author{
J. K. GOLDHABER
}

1. Introduction. The problem of arranging $v$ elements into $v$ sets in such a way that every set contains exactly $k$ distinct elements and that every pair of sets has exactly $\lambda=k(k-1) /(v-1)$ elements in common, where $0<\lambda<$ $k<v$, is equivalent to finding a normal integral $v$ by $v$ matrix $A$ such that $A^{T} A=B$, where $B$ is the $v$ by $v$ matrix having $k$ in every position on the main diagonal and $\lambda$ in all other positions (10). Utilizing the fact that for the existence of a $\lambda, k, v$ design it is necessary that $I$ (the $v$ by $v$ identity matrix) represent $B$ rationally, (2) and (3) have proved the non-existence of certain $\lambda, k, v$ designs. Neither of the proofs utilize the fact that it is necessary that $A$ be normal. However, Albert (1) for the projective plane case and Hall and Ryser (5) for the general design proved that if there exists a rational $A$ such that $A^{T} A=B$ then there exists a normal rational matrix satisfying the same equation. Thus the requirement of normality does not exclude any $\lambda, k, v$ which were not previously excluded.

It is evident that for the existence of a $\lambda, k, v$ design it is necessary that for every prime $p$ there exist an integral $p$-adic normal matrix $A$ satisfying $A^{T} A=$ $B$. Assuming that $(k, k-\lambda)=1$, we prove in $\S 2$ that if $I$ represents $B$ rationally then this necessary condition is satisfied. Thus, once again, no additional designs are excluded. It does follow, however, that if $I$ represents $B$ rationally then $I$ represents $B$ without essential denominator and, furthermore, that there is a form in the genus of $I$ which represents $B$ integrally.

In $\S 3$ we consider a modified incidence equation which is satisfied by every incidence matrix and which, if $I$ represents $B$ rationally, has integral solutions. Sufficient conditions for the existence of a $\lambda, k v$ design in terms of these integral solutions are given.

2. The incidence equation examined locally. We assume throughout this paper that $(k, k-\lambda)=1$. Thus, since $\lambda v=k^{2}-(k-\lambda)$ we have $(\lambda, k)=(\lambda, k-\lambda)=(v, k)=(v, k-\lambda)=1$. The matrices $I$ and $B$ are as above. We prove

THEOREM 1. If I represents $B$ rationally then for every prime $p$ there exists a matrix $A$ with elements in the ring $R(p)$ of $p$-adic integers such that $A^{T} A=A A^{T}$ $=B$.

Received November 3, 1958. Research supported in part by the Office of Ordinance Research under Contract DA-23-072-ORD-1051. 
We show first that there exists a matrix $C$ (not necessarily normal) with elements in $R(p)$ such that $C^{T} C=B$. It follows from well-known theorems on quadratic forms (7) and the fact that $I$ and $B$ are both positive definite that it is sufficient to show this for all primes $p \in P$, where $P$ is the set of all prime divisors of $2 \cdot \operatorname{det} B=2 k^{2}(k-\lambda)^{v-1}$. Let $T$ be the $v$ by $v$ matrix

$$
\left[\begin{array}{rrrrr}
1 & -1 & -1 & -1 & -1 \\
1 & 1 & 0 & 0 & 0 \\
1 & 0 & 1 & 0 & 0 \\
1 & 0 & 0 & 1 & 0 \\
. & . & . & . & . \\
. & . & . & . & . \\
. & . & . & . & . \\
1 & 0 & 0 & 0 & 1
\end{array}\right] .
$$

Then

$$
T^{T} T=\left[\begin{array}{cc}
v & 0 \\
0 & I_{1}+S_{1}
\end{array}\right]
$$

where $I_{1}$ is the $(v-1)$ by $(v-1)$ identity matrix and $S_{1}$ is the $(v-1)$ by $(v-1)$ matrix each of whose entries is 1. Also,

$$
T^{T} B T=\left[\begin{array}{lc}
k^{2} v & 0 \\
0 & (k-\lambda)\left(I_{1}+S_{1}\right)
\end{array}\right] .
$$

Since $(k, k-\lambda)=1, v$ is a $p$-adic unit for all odd $p \in P$. $v$ is also a 2 -adic unit in the case that $v$ is odd. Hence, for odd $p, X^{T} X=B$ is solvable in $R(p), p \in P$, if and only if $X^{T}\left(T^{T} T\right) X=T^{T} B T$ is solvable in $R(p)$; and for odd $v, X^{T} X=B$ is solvable in $R(2)$ if and only if $X^{T}\left(T^{T} T\right) X=T^{T} B T$ is solvable in $R(2)$.

We first dispose of the case when $v$ is even. Since $I$ represents $B$ rationally, $(k-\lambda)$ is a square (3); whence, obviously $T^{T} T$ represents $T^{T} B T$ in $R(p)$ for all odd $p \in P$. Furthermore, since $v$ is even and $(k, k-\lambda)=1$ it follows that $k$ and $k-\lambda$ are both odd. Thus $I$ and $B$ are properly primitive forms (that is, each has a 2 -adic unit element on its main diagonal) with unit 2 -adic determinants which, since they are rationally congruent, are congruent over the 2 -adic field. Hence ( 7 , Theorem 36 ) they are equivalent in $R(2)$. Thus, if $v$ is even $I$ represents $B$ in $R(p)$ all $p \in P$.

Suppose now that $v$ is odd. It is clearly sufficient to show that $I_{1}+S_{1}$ represents $(k-\lambda)\left(I_{1}+S_{1}\right)$ in $R(p)$ for all $p \in P$.

(i) $I_{1}+S_{1}$ represents $(k-\lambda)\left(I_{1}+S_{1}\right)$ in $R(2)$.

(a) Suppose $(k-\lambda)=2^{2 b} m$ where $b \geqslant 0$ and $m$ is odd. We make use here, and below, of the following known theorem (6):

Two improperly primitive forms (that is, each form has some 2 -adic unit element but no 2 -adic unit element on its main diagonal) in the same number 
of variables and of odd determinants are equivalent in $R(2)$ if and only if their determinants are congruent $\bmod 8$.

From this it follows that $I_{1}+S_{1}$ and $m\left(I_{1}+S_{1}\right)$ are equivalent in $R(2)$. But then, obviously, $I_{1}+S_{1}$ represents $2^{2 b} m\left(I_{1}+S_{1}\right)$ in $R(2)$.

(b) Suppose $k-\lambda=2^{2 b+1} m$ where $m$ is odd. We shall show below that in this case the assumption that $I$ represents $B$ rationally implies that $v= \pm 1$ $\bmod 8$.

If $v \equiv 1 \bmod 8$ then $I_{1}+S_{1}$ and $m\left(I_{1}+S_{1}\right)$ are both equivalent to the $\frac{1}{2}(v-1)$ fold direct sum of the matrix

$$
\left[\begin{array}{ll}
0 & 1 \\
1 & 0
\end{array}\right]
$$

Call the direct sum matrix $K$. It is thus sufficient to show that $K$ represents $2^{2 b+1} K$. Since $v \equiv 1 \bmod 8,4 \mid v-1$. Let $L$ be the $\frac{1}{4}(v-1)$ fold direct sum of

$$
\left[\begin{array}{rrrr}
0 & 1 & 0 & 1 \\
1 & 0 & 1 & 0 \\
0 & 1 & 0 & -1 \\
1 & 0 & -1 & 0
\end{array}\right] .
$$

Then $\left(2^{b} L\right)^{T} K\left(2^{b} L\right)=2^{b+1} K$ as desired.

If $v \equiv-1 \bmod 8$ then $I_{1}+S_{1}$ and $m\left(I_{1}+S_{1}\right)$ are both equivalent in $R(2)$ to $K_{1} \oplus K_{2}$. Here $\oplus$ denotes direct sum, $K_{1}$ is the $\frac{1}{2}(v-7)$ fold direct sum of

$$
\left[\begin{array}{ll}
0 & 1 \\
1 & 0
\end{array}\right]
$$

and $K_{2}$ is the 6 by 6 matrix having each entry on its main diagonal equal to 2 and all other entries equal to 1 . Note that $4 \mid v-7$. Let $L_{1}$ be the $\frac{1}{4}(v-7)$ fold direct sum of the 4 by 4 matrix given in the preceding paragraph, and let $L_{2}$ be the matrix

$$
\left[\begin{array}{rrrrrr}
-1 & 0 & -1 & -1 & -1 & 0 \\
1 & 0 & 0 & 0 & 1 & 1 \\
-1 & -1 & -1 & 0 & 0 & -1 \\
0 & 0 & 1 & 1 & 0 & 1 \\
0 & 1 & 1 & 0 & 1 & 0 \\
1 & 1 & 0 & 1 & 0 & 0
\end{array}\right]
$$

Then $\left[2^{b}\left(L_{1} \oplus L_{2}\right)\right]^{T}\left(K_{1} \oplus K_{2}\right)\left[2^{b}\left(L_{1} \oplus L_{2}\right)\right]=2^{2 b+1}\left(K_{1} \oplus K_{2}\right)$; whence, $I_{1}+S_{1}$ represents $(k-\lambda)\left(I_{1}+S_{1}\right)$ in $R(2)$ as desired.

It remains to show that if $I$ represents $B$ rationally and if $k-\lambda=2^{2 b+1} m$, $m$ odd, then $v \equiv \pm 1 \bmod 8$. Since $\lambda v=k^{2}-(k-\lambda)$ we have

$$
\left(\frac{k-\lambda}{\lambda}\right)=\left(\frac{2}{\lambda}\right)\left(\frac{m}{\lambda}\right)=1 \text {, }
$$


where $(a / b)$ is the Jacobi symbol. (Since $(k, k-\lambda)=1, \lambda$ is odd.) We thus have

$$
\left(\frac{\lambda}{m}\right)=\left(\frac{2}{\lambda}\right)(-1)^{\frac{m-1}{2} \cdot \frac{\lambda-1}{2}} .
$$

We consider the cases $b \geqslant 1, b=0$ separately.

If $b \geqslant 1$ then $\lambda \equiv v \bmod 8$. If $v=3 \bmod 8$ then $(-\lambda / m)=-1$ but this is impossible since $I$ represents $B$ rationally (3). The case $v \equiv 5 \bmod 8$ is disposed of similarly.

If $b=0$ then $\lambda v \equiv-1$ or $3 \bmod 8$ according as $k-\lambda \equiv 2$ or $6 \bmod 8$. If $k-\lambda \equiv 2 \bmod 8$ and $v \equiv 3 \bmod 8$ then $(-\lambda / m)=-1$ which is impossible. Similar easy computations exclude all possibilities other than $v \equiv \pm 1 \bmod$ 8.

(ii) $I_{1}+S_{1}$ represents $(k-\lambda)\left(I_{1}+S_{1}\right)$ in $R(p)$ for all odd $p$ such that $p \mid k$. We make use here, and below, of the following known theorem $(6,11)$. For odd $p$, two forms, $f$ and $g$, in the same number of variables and of unit determinants in $R(p)$ are equivalent in $R(p)$ if and only if

$$
\left(\frac{\operatorname{det} f}{p}\right)=\left(\frac{\operatorname{det} g}{p}\right) .
$$

The desired result is an immediate consequence of this theorem. We actually have somewhat more; namely, $I_{1}+S_{1}$ represents $(k-\lambda)\left(I_{1}+S_{1}\right)$ in $R(p)$ for all odd $p$ such that $p \nmid(k-\lambda) v$.

(iii) $I_{1}+S_{1}$ represents $(k-\lambda)\left(I_{1}+S_{1}\right)$ in $R(p)$ for all odd $p$ such that $p \mid(k-\lambda)$. Suppose $(k-\lambda)=p^{b} m$ where $(p, m)=1$ and $b>0$. We consider two cases: $(a) v \equiv 1 \bmod 4$, and $(b) v \equiv 3 \bmod 4$.

(a) Since $I$ represents $B$ rationally we must have $(v / p)=(\lambda / p)=1$, (2). Thus $\operatorname{det}\left(I_{1}+S_{1}\right)=v$ and $\operatorname{det}\left[m\left(I_{1}+S_{1}\right)\right]=m^{v-1} v$ are both units and perfect squares in $R(p)$. Therefore, $I_{1}+S_{1}$ and $m\left(I_{1}+S_{1}\right)$ are both equivalent in $R(p)$ to $I_{1}$. It is thus sufficient to show that $I_{1}$ represents $p^{b} I_{1}$ in $R(p)$. If $b$ is even, this is obvious. Suppose then that $b=2 c+1$. We use the device employed in (3). There exist integers $a_{i}, i=1,2,3,4$, such that $\sum_{1}{ }^{4} a_{i}{ }^{2}=p$. Let $L$ be the $\frac{1}{4}(v-1)$ fold direct sum of

$$
p^{c} \cdot\left[\begin{array}{rrrr}
a_{1} & a_{2} & a_{3} & a_{4} \\
a_{2} & -a_{1} & -a_{4} & a_{3} \\
a_{3} & a_{4} & -a_{1} & -a_{2} \\
a_{4} & -a_{3} & a_{2} & -a_{1}
\end{array}\right] .
$$

Then $L^{T} L=p^{2 c+1} I_{1}$ as desired.

(b) For $v \equiv 3 \bmod 4$ we must have

$$
\left(\frac{v}{p}\right)=\left(\frac{\lambda}{p}\right)=(-1)^{\frac{p-1}{2}},
$$


(3). Thus $I_{1}+S_{1}$ and $m\left(I_{1}+S_{1}\right)$ are both equivalent to the $(v-1)$ $(v-1)$ diagonal matrix $\left[1,1, \ldots, 1,(-1)^{\frac{1}{2} p-\frac{1}{2}}\right]=J$. We must show that $J$ represents $p^{b} J$. For even $b$ this is obvious and so we may take $b=2 c+1$. If $p \equiv 3 \bmod 4$ then let $L_{1}$ be the $\frac{1}{4}(v-3)$ fold direct sum of the 4 by 4 matrix given in the previous paragraph and let

$$
L_{2}=p^{c}\left[\begin{array}{ll}
a & 1 \\
1 & a
\end{array}\right]
$$

where $a$ is a $p$-adic integer such that $a^{2}=1+p$. Then $\left[\left(L_{1} \oplus L_{2}\right)\right]^{T} J\left[\left(L_{1} \oplus\right.\right.$ $\left.\left.L_{2}\right)\right]=p^{b} J$. If $p=1 \bmod 4$ then there exist integers $a_{1}$ and $a_{2}$ such that $a_{1}{ }^{2}+$ $a_{2}{ }^{2}=p$. Let $L$ be the $\frac{1}{2}(v-1)$ fold direct sum of

$$
p^{c}\left[\begin{array}{rr}
a_{1} & a_{2} \\
a_{2} & -a_{1}
\end{array}\right] \text {. }
$$

Then $L^{T} J L=p^{b} J$.

It follows from all the above that for every $p$ there exists a matrix $C$ with elements in $R(p)$ such that $C^{T} C=B$. It remains to show that there exists a normal matrix with the desired properties. If $p \nmid v$ this is clear. In fact, we have seen above that for every $p \nmid v$ there exists a $C_{1}$ with elements in $R(p)$ such that $C_{1}{ }^{T}\left(I_{1}+S_{1}\right) C_{1}=(k-\lambda)\left(I_{1}+S_{1}\right)$. Let $A=T\left(k \oplus C_{1}\right) T^{-1}$. Then $A^{T} A=B$ and $A S=k S$, where $S$ is the $v$ by $v$ matrix composed entirely of ones; whence by (5, Theorem 3.1) $A$ is normal. Since $p \nmid v, A$ has its elements in $R(p)$.

Suppose $p \mid v$. We know that there exists a matrix $C=\left(c_{i j}\right)$ with elements in $R(p)$ such that $C^{T} C=B$. Let $\alpha$ be the column vector $\left[r_{1}, r_{2}, \ldots, r_{v}\right]$ where $r_{i}=\sum_{j} c_{i j}$, and let $\beta$ be the $v$ by 1 column vector each of whose entries is $k$. We will show that there exists an orthogonal matrix $O$ with elements in $R(p)$ such that $O \alpha=\beta$. It will follow that $A=O C$ is such that $A^{T} A=B, A S=k S$; and again by (5), $A$ is normal as desired.

We use the following theorem (4, Satz 10.4). It is stated here more concretely and in a less general form that in (4).

Let $V$ be a $v$ dimensional vector space of column vectors over the $p$-adic field with a non-degenerate ground form given by the $v$ by $v$ symmetric matrix $G$. Let $\mathscr{F}$ be a lattice in $V$ and let $\mathscr{D}$ be its different. If $\alpha$ and $\beta$ are primitive vectors in $\mathscr{F}$ such that $\alpha^{T} G \alpha=\beta^{T} G \beta$ and $\alpha-\beta \in \mathscr{D}$ then there exists a $v$ by $v$ matrix $O$ with elements in $R(p)$ such that $O^{T} G O=G$ and $O \alpha=\beta$.

For our purposes we take $G$ to be the identity matrix, $\mathscr{F}$ as the lattice which has as a basis the column vectors of the identity matrix $I$, and $\alpha$ and $\beta$ as above. We note that if $p$ is odd then $\mathscr{D}=\mathscr{F}$, and if $p=2$ then $\mathscr{D}$ is the lattice which has as a basis the column vectors of $2 I$. From the fact that $C^{T} C=B$ it follows easily (10) that $\sum_{j} c_{j i} r_{j}=k^{2}$ and $\sum_{i} r_{i}{ }^{2}=k^{2} v$. From the first of the latter equations and the facts that $p \mid v,(k, v)=1$ it follows that $\alpha$ and $\beta$ are both primitive. From the second of these equations it follows that $\alpha^{T} \alpha=\beta^{T} \beta$. Hence if $p$ is odd the desired $O$ exists. 
In order to complete the proof for $p=2$ it is sufficient to show that $r_{i}{ }^{2} \equiv 1$ mod 2. Let $t_{i j}$ be the inner product of the $i$ th and $j$ th rows of $C$. Again as in (10) we have

$$
k^{2} t_{i j}=\lambda r_{i} r_{j}+k^{2}(k-\lambda) \delta_{i j} .
$$

If $r_{i}^{2} \equiv 0 \bmod 2$ then $t_{i i} \equiv 1 \bmod 2$ and we would have

$$
0 \equiv r_{i}^{2} \equiv \sum_{j} c_{i j}^{2} \equiv t_{i i} \equiv 1 \bmod 2
$$

which is clearly absurd.

This completes the proof of Theorem 1 .

As immediate consequences we have

COROLlaRY 1. If I represents $B$ over the rational field then $I$ represents $B$ rationally without essential denominator, that is, for every positive integer $m$ there is a matrix $D$ with rational elements whose denominators are prime to $m$ such that $D^{T} D=B$.

COROLLARY 2. If I represents $B$ rationally then there exists a form in the genus of $I$ which represents $B$ integrally.

3. A modified incidence equation. Since the genus of the identity contains more than one class for $v>8$ (8) Corollary 2 does not yield any new designs. It is natural, therefore, to examine a matric equation, akin to $X^{T} X=$ $B$, which is still satisfied by every incidence matrix, has integral solutions, and then to examine the relationship of these integral solutions to incidence matrices.

Theorem 2. Let $t=a / b$ be a rational number greater than $1 / v$ such that $(a v-b) b$ is odd. Let $S$ be the v by v matrix composed entirely of ones. If I represents $B$ rationally then $I-t S$ represents $B-t k^{2} S$ integrally.

For by Theorem $1, b I-a S$ represents $b B-a k^{2} S$ in every $R(p)$. (The normality of $A$ implies that $S A=A S=k S$ and therefore $A^{T}(b I-a S) A=$ $b B-a k^{2} S$.) Hence there exists a form in the genus of $b I-a S$ which represents $b B-a k^{2} S$ integrally. Since the genus of an indefinite form of odd determinant in $v>2$ variables consists of exactly one class (9) Theorem 2 follows.

Let $\mathscr{S}$ be the set of all rationals which have the properties stated in Theorem 2. For $t \in \mathscr{S}$ let $A(t)=\left(a_{i j}(t)\right)$ denote an arbitrary but fixed integral solution. of $X^{T}(I-t S) X=B-t k^{2} S$. Let $r_{i}(t)=\sum_{j} a_{i j}(t)$, and $s_{j}(t)=\sum_{i} a_{i j}(t)$.

Theorem 3: (i) If $A\left(t_{0}\right)$ is normal and $t_{0} \neq\left(k+(k-\lambda)^{\frac{1}{2}}\right) / k v$ then $A\left(t_{0}\right)$ is an incidence matrix or the negative of one.

(ii) If for $t_{1}, t_{2} \in \mathscr{S}, t_{1} \neq t_{2}$ we have $r_{i}\left(t_{1}\right)=r_{i}\left(t_{2}\right)\left(s_{i}\left(t_{1}\right)=s_{i}\left(t_{2}\right)\right)$ for $i=1,2,3, \ldots, v$, then $A\left(t_{1}\right)$ is an incidence matrix or the negative of one.

(iii) If there exists an $M$ and a subset $\mathscr{S}^{\prime}$ of $\mathscr{S}$ containing sufficiently many distinct elements (see below) such that $\left|r_{i}(t)\right|<M\left(\left|s_{i}(t)\right|<M\right)$ for $t \in \mathscr{S}^{\prime}$ and 
$i=1,2, \ldots, v$ then there exists a $t_{0} \in \mathscr{S}^{\prime}$ such that $A\left(t_{0}\right)$ is an incidence matrix or the negative of one.

(iv) If $r_{i}\left(t_{0}\right)>0$ for $i=1,2, \ldots, v$ and for $t_{0}>1$ then $A\left(t_{0}\right)$ is an incidence matrix.

(i) As in (10) the following relations may be established: For every $t \in \mathscr{S}$,

$$
\begin{gathered}
\sum r_{i}^{2}(t)-t\left(\sum r_{i}(t)\right)^{2}=k^{2} v(1-t v) \\
k^{2}(1-t v)\left(\sum s_{i}^{2}(t)\right)+\left(k^{2} t-\lambda\right)\left(\sum r_{i}(t)\right)^{2}=k^{2}(k-\lambda) v .
\end{gathered}
$$

Now the normality of $A\left(t_{0}\right)$ implies that $\sum r_{i}{ }^{2}\left(t_{0}\right)=\sum s_{i}{ }^{2}\left(t_{0}\right)$. Since $t_{0} \neq(k+$ $\left.(k-\lambda)^{\frac{1}{2}}\right) / k v$, the above equations imply that $\sum r_{i}{ }^{2}\left(t_{0}\right)=k^{2} v$ and $\sum s_{i}\left(t_{0}\right)=$ $\sum r_{i}\left(t_{0}\right)= \pm k v$. Whence $r_{i}\left(t_{0}\right)=s_{i}\left(t_{0}\right)=k$ or $r_{i}\left(t_{0}\right)=s_{i}\left(t_{0}\right)=-k$ for all $i$. But then $A^{T} A=A A^{T}=B$ and the result follows by (10, Theorem 2.1).

(ii) The proof of this result is analogous to the proof of (i).

(iii) The number of lattice points in $v$ dimensional space over the reals with components having absolute value less than $M$ is finite. Hence if $\mathscr{S}^{\prime}$ contains more elements than the number of such lattice points then there exist $t_{1}$, $t_{2} \in \mathscr{S}^{\prime}, t_{1} \neq t_{2}$, such that $r_{i}\left(t_{1}\right)=r_{i}\left(t_{2}\right)$ for $i=1,2, \ldots, v$. The desired result follows from (ii).

(iv) Once again as in (10) it may be shown that $r_{i}(t) \equiv 0 \bmod k$. Since $r_{i}(t)>0$ it follows that

$$
\left(\sum r_{i}(t)\right)^{2} \geqslant\left(\sum r_{i}^{2}(t)\right)+v(v-1) k^{2}
$$

From the first of the equation given in (i) above it follows that

$$
k^{2} v(1-t) \leqslant(1-t) \sum r_{i}^{2}(t) .
$$

Since $t>1$ we have $\sum r_{i}{ }^{2}(t) \leqslant k^{2} v$. But also

$$
k^{2} v^{2} \leqslant\left(\sum r_{i}(t)\right)^{2} \leqslant v\left(\sum r_{i}^{2}(t)\right) .
$$

Hence $\sum r_{i}{ }^{2}(t)=k^{2} v$ and the proof may be completed as was the proof of (i).

We remark that if $v>k+1$ and $t>1$ then $r_{i}(t) \neq 0$ for $i=1,2, \ldots, v$.

Theorem 3 gives sufficient conditions for the existence of a $\lambda, k, v$ design in terms of integral solutions, which by Theorem 2 are known to exist, of the matric equation

$$
X^{T}(I-t S) X=B-t k^{2} S .
$$

The problem of determining the nature of these solutions appears to be extremely difficult. Also of interest, and possibly a more pliable problem, is the determination of the integral automorphs of $I-t S$ and $B-t k^{2} S$. 


\section{REFERENCES}

1. A. A. Albert, Rational normal matrices satisfying the incidence equation, Proc. Amer. Math. Soc., 4 (1953), 554-9.

2. R. H. Bruck and H. J. Ryser, The nonexistence of certain finite projective planes, Can. J. Math., 1 (1949), 88-93.

3. S. Chowla and H. J. Ryser, Combinational problems, Can. J. Math., 2 (1950), 93-9.

4. M. Eichler, Quadratische formen und orthogonale gruppen (Berlin, 1952).

5. Marshall Hall and H. J. Ryser, Normal completions of incidence matrices, Amer. J. Math., 76 (1954), 581-9.

6. B. W. Jones, A canonical quadratic form for the ring of 2-adic integers, Duke Math. J., 11 (1944), 715-27.

7. - The arithmetic theory of quadratic forms, Carus Math. Monographs, 10 (1950).

8. W. Magnus, Ueber die Anzahl der in einem Geschlecht enthaltenen Klassen von positiv definiten quadratischen Formen, Math. Ann. 114 (1937), 465-75.

9. A. Mayer, Zürich naturf. Ges., 36 (1891), 241.

10. H. J. Ryser, Matrices with integer elements in combinational investigations, Amer. J. Math., 74 (1952), 769-73.

11. C. L. Siegel, Equivalence of quadratic forms, Amer. J. Math. 63 (1941), 658-80.

Washington University 